\title{
Lumen
}

Selected Proceedings from the Canadian Society for Eighteenth-Century Studies

\section{Courants et contre-courants dans le roman des Lumières : Célianne de Benoist}

\section{Olga B. Cragg}

Volume 16, 1997

Freedom and Boundaries

Émancipation et frontières

URI : https://id.erudit.org/iderudit/1012443ar

DOI : https://doi.org/10.7202/1012443ar

Aller au sommaire du numéro

Éditeur(s)

Canadian Society for Eighteenth-Century Studies / Société canadienne d'étude du dix-huitième siècle

ISSN

1209-3696 (imprimé)

1927-8284 (numérique)

Découvrir la revue

Citer cet article

Cragg, O. B. (1997). Courants et contre-courants dans le roman des Lumières : Célianne de Benoist. Lumen, 16, 103-112. https://doi.org/10.7202/1012443ar d'utilisation que vous pouvez consulter en ligne. 


\section{Courants et contre-courants dans le roman des Lumières: Célianne de Benoist}

Cette étude vise à éclairer la modernité d'un petit roman intitulé Célianne ou les amans séduits par leurs vertus, ${ }^{1}$ publié en 1766 par un auteur jusqu'à présent aussi peu connu que ses oeuvres, Françoise-Albine Benoist. ${ }^{2} \mathrm{Ce}$ texte, que l'auteur désigne dans sa préface comme simple «Brochure», se situe en plein milieu des divers courants romanesques caractéristiques du genre romanesque de la seconde moitié du dix-huitième siècle. Benoist est l'auteur d'au moins cinq autres romans: Elisabeth, paru la même année que Célianne, les Lettres du colonel Talbert (1767), Agathe et Isodore (1768) et l'Erreur des désirs (1770), sans compter d'autres textes relevant de la littérature dite "personnelle» (Van Dijk 231) et d'articles parus dans le Journal des Dames. Elle a publié Célianne, récit ramassé d'environ 200 pages, cinq ans après la sortie de La Nouvelle Héloïse. Alors que la plupart de ses romans sont livrés en 4-5 tomes ou 800 pages in-douze, Célianne se distingue par sa brièveté.

Dans un premier temps, nous verrons en quoi le roman se rattache à la tradition romanesque rousseauiste, puis dans un deuxième temps, nous analyserons les jeux formels expérimentaux repérables dans le texte, en mettant l'accent sur la problématique des rapports entre la narratrice, l'héroïne et le lecteur. On verra comment Benoist met en cause certaines conventions du roman - la complicité entre auteur et lecteur, par exemple - rejoignant par là les innovateurs du genre romanesque tels que Laurence Sterne et Diderot.

$\mathrm{D}^{\prime}$ abord, résumons brièvement l'essentiel du récit, dont les grandes lignes sont indiquées dans le premier paragraphe de la préface. La perspective morale de l'auteur sort du chemin ordinaire selon ellemême, puisque le dénouement vertueux est contraire aux moeurs telles qu'elles sont souvent représentées dans les romans libertins de l'époque. L'éditeur fictif de la préface, anticipant la critique du roman, cherche à mettre le lecteur dans son camp. Ainsi, à partir d'un plaidoyer auprès du lecteur, dès les premières lignes du roman, un dialogue va s'établir entre narratrice et narrataire en vue de s'allier celui-ci: Qu'on «ne se hâte 
[hâtent] point de condamner l'Auteur, avant d'examiner quel a pu être son motif en traitant un Chapitre aussi délicat, \& l'on peut ajouter aussi incroyable que celui d'un amour honnête entre des personnes engagées» (vii). L'éditeur fictif se propose donc d'étudier, d'un point de vue moral, le danger d'une amitié qui devient trop intime entre deux personnes qui s'aiment d'abord innocemment et veulent avant tout préserver leur réputation. Célianne, femme mariée à un homme que ses parents lui ont choisi, végète dans une existence vide où l'opéra, les bals, et les promenades sont les seules occupations possibles. Sa vie dénuée de passion et de sentiments ne lui apporte que désespoir, la confirmant dans son idée qu'elle n'a jamais aimé véritablement. Il n'est pas surprenant que ses devoirs d'épouse modèle respectueuse de son époux ne puissent combler ses aspirations. Notre héroïne voudrait aimer vraiment «pour avoir des sacrifices à faire à la vertu", comme on le lit dans le Mercure de France (août 1766, 151) au sujet de l'hérö̈ne. Son mari lui présente Mozime, un jeune célibataire modeste, respectueux, sensible, et doux. Un envoûtement inévitable s'ensuit, d'abord marqué par une amitié pure et désintéressée qui les pousse à passer tout leur temps ensemble. Ils sont persuadés qu'un goût partagé pour la vertu et le désir mutuel de la pratiquer les unissent, mais en fait, comme on peut s'y attendre, ils se leurrent dans l'interprétation de leurs sentiments, et glissent vers un piège fatal: Mozime, sur le point de succomber à sa passion, aurait ravi sa vertu à Célianne sans la fâcheuse interruption du mari.

Mariage arrangé, jalousie, malentendus, missives explicatives, lettres perdues, fausse mort, périls de la séduction, désillusions sur la nature véritable des sentiments, et exil de Mozime - tels sont les topoï d'une intrigue qui relève du roman sentimental. Après une absence d'une année, le héros, se conformant aux voeux de sa famille et de Célianne, revient, maintenant marié, partager les douceurs de l'amitié à quatre:

Ils ne purent se revoir sans émotion; mais trop vertueux l'un et l'autre pour se livrer à de coupables sentiments, ils les renfermerent dans leur coeur, et passèrent le reste de leur vie dans la plus pure et la plus parfaite amitié; bonheur dont ils n'auroient pas jouï, s'ils se fussent livrés à leurs criminels désirs. (223; je souligne)

Cette clôture optimiste, caractéristique du roman sentimental de l'époque est soulignée par la terminologie d'usage: vertueux, coeur, amitié, bonheur, désirs invite à classer le roman dans le domaine de l'idéalisme à la Rousseau.

Le code romanesque patriarcal, qui préconise le mariage de convenances comme réussite sociale pour la femme comme d'ailleurs pour l'homme, et que Benoist semble approuver, est doublé d'une voix moralisatrice féminine qui parle certaines valeurs et vérités psychologiques 
prônées spécifiquement par les femmes selon la narratrice, et qui sont à distinguer des idées de Rousseau. Benoist se situe elle-même dans la lignée des romanciers prédicateurs dont la portée morale et sociale semble de première importance. «Démontrer l'inévitable danger d'une liaison conçue par un coeur novice, et formée sous les auspices de l'innocence, est le but qu'on s'est proposé» (viii), écrit-elle dans la préface. De surcroît, elle élargit son sujet pour valoriser l'effet de la conduite des femmes sur toute la nation, selon elle. Le platonisme initial $\mathrm{du}$ couple Célianne-Mozime, derrière lequel cependant se cache un érotisme latent, représente un piège dangereux qui peut aboutir au désordre typique à tous les niveaux du groupe social: «Le repos des Citoyens, le bonheur des familles et l'harmonie de la société en dépendent» (viii). Remarquons au passage la place capitale qu'elle attribue aux rapports interpersonnels intimes, comme base fondamentale de la société.

La préface du roman se présente comme un discours pédagogique où l'éditeur fictif joue le rôle de conseiller averti pour mettre à nu la crise de l'âme que traverse une femme mariée contre son gré à un homme qui lui est souvent indifférent, voire qu'elle trouve repoussant. Il s'agit d'éclairer une femme vertueuse, naïve, inexpérimentée, et incapable de déjouer les véritables intentions et les manoeuvres d'un séducteur dont le mérite apparent représente le plus grand appât pour cette sorte de femme sensible. L'avant-dernier paragraphe de la préface résume avec force l'avertissement contre la fatale évolution d'une passion aveugle. Benoist s'ingénie à communiquer ce message à la femme vulnérable, aliénée par une éducation négligée, mais pour qui le devoir conjugal symbolise tout de même un certain sentiment de satisfaction grâce au prestige social accordé à l'épouse dévouée:

S'en occuper [de l'homme méritoire] est un plaisir qu'on se croit permis; le voir, est un besoin qu'on se dissimule; lui donner des marques d'estime, est une jouissance qu'on regarde comme innocente; en être séparée, est une privation insupportable: insensiblement on parvient au comble du prestige, \& on ne voit l'abîme que lorsqu'on y est plongé. (xv; je souligne)

On suit dans ce passage la progression du désir aveugle vers sa fin destructrice, grâce à une série de verbes placés au début de chaque segment de phrase et qui martèlent la fatalité de ce rapport en apparence innocent. La vérité de l'expérience humaine prend corps dans celle du langage.

A l'instar de Rousseau, il se dégage de Célianne une voix professorale mais qui parle sur le mode mineur réaliste. Les conseils de l'auteur se révèlent par des stratégies patriarcales telles que la maxime et les 
énoncés sentencieux. Le nombre de déclarations généralisantes dans ce texte est exceptionnellement élevé pour une oeuvre aussi courte. Les manifestations du discours moral de Benoist ont en commun le recours aux lois psychologiques et éthiques liées à la situation relationnelle entre Célianne et Mozime. La proposition d'ordre général qui apparaît comme une "phrase-noyau» est dans l'ensemble un enchaînement des causes et des effets de la trame événementielle. La maxime qui cristallise la destinée de la femme au dix-huitième siècle acquiert une fonction utilitaire dans le sens qu'il y a une complicité entre le sujet et la force extérieure qui pousse la victime à se cloîtrer dans un mariage indifférent. C'est le cas de la maxime suivante: «Quand on prend le parti de l'obéissance, on dédaigne de veiller à sa destinée» (4), écrit Benoist à propos de la conduite de Célianne lorsque celle-ci s'abandonne à la loi du père quant au choix de son époux.

Les vérités mises en lumière dans Célianne correspondent souvent aux thèmes privilégiés par le lexique utilisé par Benoist, comme on le voit dans la série: pureté des sentiments, amour, imagination, vice, déguisement, plaisir, amour-propre. La visée pédagogique associée à ces notions se traduit par des observations psychologiques tirées de la conduite instinctive des personnages en situation, ainsi par exemple:

Se croire essentielle à quelqu'un qu'on honore de sa plus haute estime, est un appas si flatteur pour l'amour-propre, qu'il conduit bientôt et imperceptiblement à un engagement plus tendre entre personnes de sexe différent. (12)

Envisagée sous l'angle pragmatique, cette maxime proleptique signale le chemin dangereux que pourra prendre une amitié innocente pour les personnages du roman; en dehors du contexte narratif, cependant, l'avertissement moral devient un message destiné au lecteur/lectrice.

$\mathrm{Au}$ risque de multiplier les exemples, passons à une autre sorte de doxa - c'est-à-dire à des énoncés qui relèvent de la phrase à sens général - la citation latine ${ }^{3}$ qui entretient cette image de l'homogénéité systématique du monde statique moral. Même si l'utilisation parodique des tournures classiques - ici dans un contexte médical - semble inattendue, elle renforce le sens d'autorité qui domine la société patriarcale de l'époque. L'épisode de la maladie de Célianne, qui prend des allures satiriques, illustre l'attitude moqueuse de l'auteur à l'égard de la domination masculine. Les neuf médecins sollicités ont des noms aussi absurdes que dérogatoires - Dandelin, Flanquin, Cremone, Raphin (rat fin), Marmeli, Civette, Bloquet, Carmel, et Clichet. Ils sont là pour guérir Célianne d'une maladie qu'ils attribuent à un malaise féminin dû à une cause peu scientifique, paraissant ainsi juger de son état selon une formule rigide, typique du préjugé: 
C'est avec raison qu'Aristote prétend que les affections de l'âme sont seules la source des infirmités de l'homme. Cette hypothèse est encore plus vraie par rapport à la plus belle portion de l'humanité: les femmes ont le coeur plus affectueux et plus tendre. Ce penchant qu'on leur voit tous les jours manifester pour des serins, des angola, des épagneuls et des singes; le chagrin qu'elles témoignent, les évanouissement qu'elles éprouvent lorsqu'elles viennent à perdre les objets de leur tendresse, est une preuve non équivoque que les femmes en général n'ont jamais de maladies plus dangereuses que quand elles ont eu le sang troublé par quelques-uns de ces fâcheux événemens, auxquels la bonté de leur coeur les rend très-sensibles. (165)

Les digressions et les préceptes à base doctrinale, exprimés par les multiples phrases endoxales, sont amplifiés par des échos intertextuels de Montaigne, Solon, Erasistrate, et Aristote ${ }^{4}$, et tissent ainsi une toile de propositions éthiques et psychologiques, mais colorée d'un ton franchement moqueur.

Cette abondance de formes brèves au caractère moral et généralisant - sentences, aphorismes, maximes - répond au goût persistant au dix-huitième siècle pour l'édification selon Labrosse: «La recherche de l'instruction et du précepte ne signale pas seulement chez ces lecteurs une fascination pour la vertu ou une inquiétude morale, elle constitue aussi un profond réflexe culturel» (44). Comme nous l'apprennent les inventaires des bibliothèques privées, les lecteurs de l'époque des Lumières sont avides de lire, par exemple, des moralistes comme La Bruyère, La Rochefoucauld, Chamfort, et Vauvenargues.

Il n'est pas surprenant d'apprendre qu'à la parution de ce second roman de Benoist, pas moins de sept périodiques de l'époque se sont intéressés à cette oeuvre. ${ }^{5}$ Le compte rendu paru dans le Mercure de France (août 1766) était le plus enthousiaste et le plus étendu - il avait une dizaine de pages. Mais malgré cette réaction favorable, le roman a vite sombré dans l'oubli. Il faut nous demander pourquoi?

Etrangement, ce qui a peut-être rebuté les premiers lecteurs de Benoist, peut aujourd'hui être relu à la lumière de la théorie de la réception. C'est que le style littéraire de Célianne, qualifié de «maniéré» et peu naturel dans l'Année littéraire, révèle des techniques narratives restées inaperçues jusqu'ici et que le concept de l' «horizon d'attente» de Hans Jauss (46) permet de mettre en évidence. Ainsi, précisément, les commentaires éditoriaux qui ont pu autrefois ralentir la lecture, comme on le voit dans le Journal des Savants, deviennent pour nous intéressants: "[L]es notes sont la partie faible de ce modèle admirable . . . elles troublent par des plaisanteries ou des réflexions déplacées l'intérêt que l'ouvrage inspire, cependant elles ne sont point à dédaigner», lit-on dans le numéro de décembre 1766. Un tel jugement fait apparaître la distance 
critique qui nous sépare du dix-huitième siècle. Le fait que cette partie auto-référentielle du texte sous forme de notes infrapaginales ait été relevée est en soi significatif: il souligne l'importance de ces éléments dans le texte. C'est justement cette présence, ce regard critique de la narratrice qui rend ce petit roman exceptionnel. Par comparaison, un recensement des grands axes thématiques des douze périodiques qui ont accueilli La Nouvelle Héloïse en 1761 n'indique aucun relevé des fonctions des notes (Labrosse 138-139).

Ce qui distingue donc particulièrement Célianne, roman dit sentimental, de l'abondante production romanesque de cette époque, c'est sa structure expérimentale. Le texte, par la double présence de la narratrice dans le récit/discours et dans les notes infrapaginales, joue avec virtuosité de divers effets narratifs: distanciation, ironie, niveaux des commentaires. Cette fonction de la narratrice comme personnage fictif dépasse la limite traditionnelle de la perspective objective d'un organisateur intradiégétique du texte, quoique la narratrice joue ce rôle intradiégétique elle aussi. La récurrence notable de la technique de l'annotation - ici vingt-quatre notes marginales, plus des interpellations au lecteur qu'elles contiennent et que l'on retrouve également à l'intérieur de la trame diégétique - souligne l'aspect formel non conformiste de Célianne. Il est à noter que la longueur de ces remarques varie considérablement, allant de la plus courte, d'une trentaine de mots, à la plus longue qui dépasse 130 mots.

Ces notes illustrent d'abord la fonction dite testimoniale du rapport entre la narratrice/personnage et l'héroïne, situées toutes les deux sur le même plan malgré les paroles extradiégétiques de la narratrice. La note treize, par exemple, illustre clairement un mouvement circulaire traversant les frontières de la fiction et de la méta-narration. La narratrice s'identifie en quelque sorte à son personnage dans la mesure où elle prétend connaître personnellement l'héroïne et où elle l'interpelle sur un ton moqueur:

Eh! Ne le voyez-vous pas, trop aveugle Célianne, ce qu'il falloit faire. C'étoit de bannir Mozime, de le fuir vous-même: mais ce parti eût trop coûté à votre tendresse; il vous fut bien plus facile d'immoler l'idole de votre vanité que celle de votre coeur. (89)

Cette note extradiégétique s'adresse apparemment à un double destinataire: d'abord la narratrice parle à Célianne pour la réprimander; ensuite la lectrice naïve et innocente est implicitement invitée à mieux choisir dans la vie réelle une conduite plus digne que celle de l'héroïne. De toute évidence, les intentions didactiques font partie intégrante du dialogue entre la narratrice et l'héroïne, d'un côté, entre elle et le lecteur, 
de l'autre. Les nombreuses interpellations au lecteur impliquent son adhésion au but édificateur. En effet, la tentative de communication d'un message didactique ponctue le texte régulièrement, dans des phrases telle que: «Mais revenons aux preuves que j'ai promis de cette fâcheuse vérité» (31). Ici la narratrice s'adresse à son lecteur. Une telle familiarité ambiguë entre narrateur, héroïne, et lecteur donne l'impression d'un flou dans la focalisation du roman.

La confusion qui en résulte provient de l'omniprésence de la narratrice qui se manifeste entre les différents plans du récit. Par exemple, l'aveu que Célianne est une amie ajoute une nouvelle dimension à l'existence de la narratrice: «Elle m'a fait si souvent des confidences à ce sujet ... ». (31). Le pronom à la troisième personne, désignant Célianne, révèle le rapport intime entre l'héroïne $\mathrm{d}^{\prime}$ une part, et la narratrice-personnage devenue actrice dans le roman de l'autre. Cette entrée insolite joue un rôle crucial. La narration du récit prend ainsi un ton ironique du fait de la répétition insistante de pronoms possessifs tels que «mon héroïne», «mes héros» ou "mon héros», et du démonstratif "cette tendre Amante». Si la narratrice produit un effet de distanciation du point de vue esthétique, son ambivalence n'en est pas moins capitale pour la vision que le lecteur a de son propre rôle dans l'univers romanesque. La distanciation ironique apparaît aussi lorsque l'éditeur, c'est-à-dire la narratrice comme historienne plutôt que romancière, déjoue et inverse par exemple la notion du portrait conventionnel de l'héroïne idéale aux cheveux blonds:

Ils [les cheveux] n'étoient cependant pas blonds, comme on les suppose à toutes les héroïnes de Romans. Je ne pourrai pas même les comparer au jais, à l'ébène, au fil de l'or. Pour dire la vérité, comme tout Historien le doit, je dirai qu'ils étoient châtains-clairs. (84)

Ce type d'intervention revient à nier les conventions romanesques de l'époque. En 1766, une telle remise du genre romanesque doit être qualifiée pour le moins d'inattendu.

La fonction explicative et corrective des notes de la narratrice est peut-être la plus ordinaire. Elle s'illustre quand elle ajoute un trait psychologique au portrait en mouvement qu'elle est en train de construire de son héroïne, pour ne pas alourdir le récit. Mais d'autre part, pour éclairer l'état émotif de l'hérö̈ne avec moquerie, la narratrice a recours au commentaire extradiégétique: «Il faut convenir que jamais femme ne fut plus ingénieuse à se tromper elle-même, tout en croyant de bonne foi ne travailler que pour la vertu» (91). Par ailleurs, le rôle de la narratrice se transforme parfois en celui de metteur en scène, lorsqu'elle orchestre les gestes et la conduite de ses personnages. ${ }^{6}$ 
Elle dialogue avec son lecteur pour l'entraîner à examiner la conduite de ses personnages d'un regard plus sévère. Les notes infrapaginales commencent souvent par des expressions cherchant l'adhésion du lecteur: «il faut convenir que . ..» (91); «il n'est pas inutile de remarquer . . .» (19); «il faut avouer qu'elle ...» (217). Le souci didactique de certaines des notes est manifeste dans les maximes généralisantes du type: «Voilà comme, dans plusieurs circonstances, un orgueil déplacé force à ramper, après avoir fait parade d'une vaine et ridicule fierté» (169). Le rapport que la narratrice entretient avec l'histoire racontée est, la plupart du temps, centrée sur l'évaluation des actions des personnages. "Il vaut mieux vaincre ... il lui auroit mieux convenu»: de telles phrases expriment le jugement de la narratrice.

Parmi la douzaine de rôles possibles que peut remplir la narratrice chez Benoist, les fonctions d'annotateur et de commentateur prédominent. Il s'agit pour elle de guider le lecteur et de le mettre sur la bonne voie, c'est-à-dire de l'induire à la «bonne lecture» du texte. L'intention est donc d'établir une perception autre que celle du protagoniste et d'introduire une distance entre lui et le lecteur, c'est-à-dire de le forcer à juger et à lire d'une façon critique. L'introduction d'un regard objectif sur le récit se remarque par le dialogue entre la narratrice et le lecteur. Il s'établit aussi un jeu taquin, un peu à la manière de Jacques dans le roman de Diderot, entre narratrice et lecteur. La phrase suivante peut servir d'illustration de ce procédé ludique:

Lecteur desireroit peut-être aussi sçavoir quelles étoient les réflexions de Mozime; mais il faudroit les tirer de mon imagination si je voulois satisfaire sa curiosité à cet égard, parce que les hommes en amour ne réfléchissent, et ne causent point tant avec eux-mêmes que les femmes. (47)

La narratrice avoue là son incapacité de tout savoir pour se limiter seulement à la vérité connue en tant que femme.

En conclusion, Célianne, avec ses digressions et ses expressions endoxales qui tournent autour du précipice à éviter, c'est-à-dire la préservation de la vertu féminine, appartient clairement au courant dominant des années soixante du dix-huitième siècle, courant qui sollicite une lecture morale centrée sur la problématique de l'amitié idéale, ainsi que Labrosse l'a constaté au sujet de La Nouvelle Héloïse: «La stratégie de la lecture s'articule sur le phénomène de la chute, comme si le roman à message devait toujours tourner autour d'une corruption ou d'une brisure» (55). Il semble bien que ce jugement s'applique également au roman de Benoist.

Comme on l'a vu, les techniques narratives de l'auteur transgressent pourtant les anciennes pratiques romanesques en mettant en cause la 
vraisemblance du roman traditionnel grâce à la présence d'une narratrice taquine et d'un lecteur censé actif dans sa lecture. L'originalité de cette oeuvre se situe également dans les nombreuses digressions qui suggèrent des jeux subtils entre lecteur, personnage fictif, et lecteur réel. Ce dernier lecteur remarquera surtout une certaine modernité dans ce roman aujourd'hui oublié. On peut lire dans le premier livre de Tristram Shandy:

Digressions, incontestably, are the sunshine; - they are the life, the soul of reading! - take them out of this book, for instance, - you might as well take the book along with them. (229)

Est-ce une coïncidence que se retrouvent chez Benoist et Sterne ces affinités pour la rupture et les digressions? Double présence de la narratrice et du lecteur, moquerie/ironie du ton chez elle rappellent étrangement l'auteur anglais. N'oublions pas que dans les années précédant la publication de Célianne, la présence littéraire et physique de Sterne en France était phénoménale. ${ }^{7}$ Il ne serait pas étonnant que Benoist ait lu des comptes rendus du roman de Sterne dans des périodiques français, par exemple celui qui parut dans le Journal Encyclopédique (mai, 1761) où étaient relevés certains des procédés narratifs et stylistiques de Sterne. On a l'habitude de tendre en ligne droite la chaîne des innovations romanesques de Sterne à Diderot. Il semblerait cependant que les tentatives narratologiques de Benoist forment un maillon féminin négligé et cependant non négligeable de cette chaîne de l'évolution du genre romanesque.

OLGA B. CRAGG

University of British Columbia

\section{Notes}

1 Françoise-Albine Puzin de La Martinière, madame Benoist, Célianne ou les amans séduits par leurs vertus (Amsterdam \& Paris: Lacombe, 1766). C'est la seule édition qui existe de ce roman. Je vais me référer à ce roman simplement par Célianne.

2 La critique semble avoir négligé son oeuvre. Mentionnons une thèse allemande (Krings 1982), quelques pages probantes de Joan Hinde Stewart et de Suzanna Van Dijk.

3 Des six phrases latines qui se trouvent dans le roman, cinq appartiennent à la catégorie de maximes. La phrase «Sperne voluptates, nocet empta dolore voluptas» (161) dans un latin vraiment corrompu, se traduit approximativement 
par «Se tenir loin de la volupté; la volupté prise à douleur est funeste». Ici le lien qu'un médecin, figure d'autorité masculine, établit entre le plaisir et la maladie pour une femme, est présenté comme un précepte absolu.

4 Ces noms paraissent dans le texte.

5 Affiches, Annonces et Avis divers (janvier 21, 1766); Année littéraire, II (1766); Mémoires secrets dits de Bachaumont (juin 30, 1766); Journal des Savants (décembre 1766); Correspondance Littéraire (mai 1766); Journal des Dames (mai 1766); Mercure de France (août, 1766). Voir aussi Van Dijk 256.

6 "Il est bon de remarquer que pendant tout ce discours, Mozime avoit les yeux tendrement fixés sur ceux de Célianne; qu'ils n'avoient cessé l'un et l'autre de se serrer les mains» (54). C'est une note de l'éditeur en bas de page.

7 L'intérêt pour Tristram Shandy s'accrut encore lors des voyages de Sterne en France (1762-64 et 1765) (Barton 6).

\section{Textes cités}

Barton, Francis Brown. Laurence Sterne en France. Paris: Hachette, 1911.

Benoist, Françoise-Albine Puzin de La Martinière. Celianne ou les amans séduits par leurs vertus. Amsterdam et Paris: Lacombe, 1766.

Jauss, Hans Robert. Pour une esthétique de la réception. Paris: Gallimard, 1978.

Krings, M. M. Madame Benoît, eine «romancière oubliée» des 18. Jahrunderts. Thèse. Aix-laChapelle, 1982.

Labrosse, Claude. Lire au XVIIle siècle: «La Nouvelle Héloïse» et ses lecteurs. Lyon: Presses universitaires de Lyon, 1985.

Sterne, Laurence. Tristram Shandy. Ed. Robert Maynard Hutchins. Great Books of the Western World. Chicago: Chicago UP, 1952.

Stewart, Joan Hynde. Gynographs: French Novels by Women of the Late Eighteenth Century. Lincoln and London: U of Nebraska P, 1993.

Van Dijk, Suzanna. Traces de femmes, Présence féminine dans le journalisme française du XVIIle siècle. Amsterdam et Maarsen: Holland UP, 1988. 